\section{P1-389 COMMON MENTAL DISORDERS AMONG ELDERLY INDIVIDUALS: PREVALENCE AND ASSOCIATED FACTORS}

doi:10.1136/jech.2011.142976f.80

F Borim, M Barros.* State University of Campinas, Campinas, São Paulo, Brazil

Introduction The elderly population in Brazil had grown significantly in recent decades. Brazilian studies involving adults report a high prevalence of common mental disorders (CMDs). The aim of the present study was to analyse the prevalence of CMDs (assessed using the SRQ-20) and associations with socio-demographic variables, health-related behaviour and illness among elderly individuals. Methods A population-based cross-sectional study was carried out with two-stage conglomerate sampling, using data from a home survey carried out in Campinas, SP, Brazil in 2008/2009. Prevalence values were estimated and prevalence ratios were calculated and adjusted using Poisson regression. Data analysis considered the weights related to the sampling design.

Results The data from 1518 individuals aged 60 years or more were analysed. The prevalence of CMDs was $15.5 \%(12.9 \%-18.5 \%)$. Significantly higher prevalence values were found among women, older individuals, those with no occupation, sedentary individuals, those who consumed fruit and vegetables less than four times a week and those with a poorer self-assessment of health. The prevalence value increased significantly with the increase in reported illnesses. All diseases investigated were associated to CMDs, with the exception of asthma/emphysema. The conditions with the strongest associations to $\mathrm{CMDs}$ were rheumatism $(\mathrm{PR}=2.22$; 1.73-2.83) and tendonitis ( $\mathrm{PR}=2.22 ; 1.57-3.21)$.

Conclusion The findings of the present study contribute information for guiding the planning of interventions directed at health promotion for the elderly.

\section{P1-390 EARLY AND CONTEMPORARY DETERMINANTS OF COGNITIVE FUNCTION}

doi:10.1136/jech.2011.142976f.81

A Barros, ${ }^{*}$ I Santos, A Matijasevich. Federal University of Pelotas, Pelotas, Rio Grande do Sul, Brazil

Introduction The 2004 Pelotas (Brazil) Birth Cohort has put strong emphasis on child development and cognition. In this work we explore how early and contemporary determinants relate to cognitive ability measured by WPPSI IQ test at age four.

Methods Early determinants explored were wealth, maternal schooling, smoking during pregnancy, birth size and neonatal morbidity, based on information collected just after birth and at 3 , 12 and 24 months of age. Contemporary determinants, assessed at age four, were child stimulation and malnutrition. Interviews were done by specially trained interviewers, the WPPSI test was done by psychologists that underwent a specific training. Analyses were carried out using IO as a continuous standardised variable through linear regression models adding fractional polynomials when nonlinear relationships were detected.

Results IQ mean was 100 , with s.d. of $16.5 ; 3.4 \%$ of 3723 children assessed were below 70. Maternal schooling and wealth had independent and linear effects on IO. Children from mothers $0-4$ years of schooling were one full s.d. below children from graduate mothers. Children in the poorest wealth quintile presented an average IQ $-0.5 \mathrm{SD}$ compared to the richest. Birth weight presented different effects for children small (SGA) and adequate for gestational age, negligible effect in the latter group, but for SGA the effect was strong and non-linear with low birthweight children up to -1.7 SD below normal children. Children poorly stimulated had $0.5 \mathrm{SD}$ disadvantage.
Conclusion Child IQ has complex biological and social determinants, reducing the disadvantage of vulnerable children requires integrated and coordinated action.

\section{P1-391 NO EXCESS MORTALITY IN PATIENTS OF 50 YEARS AND OLDER WHO RECEIVED TREATMENT FOR CARCINOMA IN SITU OF THE BREAST}

doi:10.1136/jech.2011.142976f.82

${ }^{1} \mathrm{E}$ Bastiaannet, ${ }^{*}{ }^{1} \mathrm{~W}$ van de Water, ${ }^{1} \mathrm{R}$ Westendorp, ${ }^{2} \mathrm{M}$ Janssen, ${ }^{1} \mathrm{C}$ van de Velde, ${ }^{1} \mathrm{~A}$ de Craen, ${ }^{1} \mathrm{G} \mathrm{J}$ Liefers. ${ }^{1}$ LUMC, Leiden, The Netherlands; ${ }^{2} \mathrm{CCCS}$, Eindhoven, The Netherlands

Background The incidence of breast carcinoma in situ has increased at a fast rate. Most clinical series have focused on cancer recurrence, rather than risk of death per se. Aim of this study was to assess the incidence and treatment in the Netherlands and estimate the excess mortality risk of carcinoma in situ.

Methods From the Netherlands Cancer Registry, adult females with carcinoma in situ were selected. Treatment was assessed according to age. Relative mortality at 10 years of follow-up was calculated by dividing observed mortality over expected mortality and stratified for age and treatment. Expected mortality was calculated using the Dutch general population matched by age and year

Results Overall, 8879 patients were included in this study. For patients aged 50-64 and 65-74 an increase in breast conserving surgery was observed over time $(p<0.001)$. For patients over 75 years of age, $8.0 \%$ did not undergo surgery. For all ages adjuvant radiotherapy after breast conserving surgery increased. For patients who received no surgery, observed mortality exceeded expected mortality in all ages (excess ratio ranged from 2.5 to 8.7). Overall, treated patients aged 50 years and older experienced no excess mortality due to carcinoma in situ regardless of treatment (ratio of 1.0, 0.96 and 1.0, respectively).

Conclusion The present population-based study of almost 9000 patients showed no excess mortality in surgically treated women over 50 years with carcinoma in situ. The estimated risk of death from breast cancer within 10 years following diagnosis was low with every (surgical) treatment option.

\section{P1-392 THE EVALUATION OF A NOVEL GENERAL POPULATION SAMPLING FRAME: AN ONLINE SOLUTION FOR A PERSISTING PROBLEM?}

doi:10.1136/jech.2011.142976f.83

${ }^{1} \mathrm{~N}$ Basu, ${ }^{1} \mathrm{~L}$ Swafe, ${ }^{2} \mathrm{D}$ Reid, ${ }^{1} \mathrm{G}$ Macfarlane, ${ }^{1} \mathrm{G}$ Jones. ${ }^{1}$ University of Aberdeen, Epidemiology Group, Institute of Applied Health Sciences, Aberdeen, UK; ${ }^{2}$ University of Aberdeen, Division of Applied Medicine, Aberdeen, UK

Introduction Due to increasingly restrictive privacy regulations, it has become difficult to access representative general population samples. In the UK, commercially available online datasets are available that pool data from several sources, but are generally untested in terms of completeness and accuracy.

Aim To evaluate the performance of a commercially available online dataset (192.com) as a population sampling frame.

Methods Firstly, 192.com was employed to identify age- and sexmatched population controls for a case-control study. Selected persons were sent a health questionnaire. Secondly, 192.com was challenged with a sample of consecutive hospital attendees whose NHS data allowed testing for coverage and accuracy. Population representativeness was assessed by comparing demographic characteristics of persons who were/were not identifiable via 192.com, using simple descriptive statistics. 